\title{
Principal directions in a gravitational field
}

\author{
By A. G. D. Watson. \\ Communicated by Professor H. W. Turnbull. \\ (Received 27th May, 1938. Read 4th June, 1938.) \\ (Received in revised form 5th September, 1938.)
}

I. In a general metric space of four dimensions, with an interval given by $-d s^{2}=g_{\mu \nu} d x^{\mu} d x^{\nu}$, where $g=\left\|g_{\mu \nu}\right\|<0$, we can choose locally galilean coordinates at any point. The initial directions of the axes can be fixed in an absolute fashion as the directions of the principal axes of the quadric $G_{\mu \nu} d x^{\mu} d x^{\nu}=$ const., where $G_{\mu \nu}$ is the contracted Riemann-Christoffel tensor.

In a gravitational field, however, at a point in empty space, $G_{\mu \nu}=\lambda g_{\mu \nu}$, and the quadric degenerates, so that this procedure breaks down.

Now the Riemann-Christoffel tensor has 20 independent components. The equations $G_{\mu \nu}=\lambda g_{\mu \nu}$ impose 10 conditions on these, so that 10 independent components still remain. We shall show here that these 10 quantities can be used, in general, to define absolute coordinate directions at any point. There is in fact, in general, an alternative method of fixing absolute directions to the use of the quadric of curvature, which remains valid when that quadric degenerates.

II. Two simple transformations, preserving the galilean character of coordinates, namely a rotation about an axis and a Lorentz transformation for motion in the direction of the same axis, are evidently commutative and can be combined into one. If $\theta$ be the angle of the rotation and $\tanh \phi$ the velocity of the motion, we have, for the combined transformation,

$$
\left.\begin{array}{l}
x_{1}=x_{1}{ }_{1} \cosh \phi+x_{{ }_{1}} \sinh \phi \\
x_{2}=x^{\prime}{ }_{2} \cos \theta-x^{\prime}{ }_{3} \sin \theta \\
x_{3}=x_{3}^{\prime} \cos \theta+x^{\prime}{ }_{2} \sin \theta \\
x_{4}=x^{\prime}{ }_{4} \cosh \phi+x^{\prime}{ }_{1} \sinh \phi
\end{array}\right\},
$$


which can also be written,

$$
\begin{aligned}
& x_{1}=x_{1}^{\prime} \cos i \phi-i x^{\prime}{ }_{4} \sin i \phi \\
& x_{2}=x_{2}^{\prime} \cos \theta-x_{3}^{\prime} \sin \theta \\
& x_{3}=x_{3}^{\prime} \cos \theta+x_{2}^{\prime} \sin \theta \\
& x_{4}=x^{\prime}{ }_{4} \cos i \phi-i x_{1}^{\prime} \sin i \phi
\end{aligned}
$$

Any transformation which is permitted can be made up of three transformations of this type taken in a determinate order.

Consider now the behaviour of the surface-element

$$
d S^{\mu \nu}=d x^{\mu} \delta x^{\nu}-d x^{\nu} \delta x^{\mu}
$$

under the transformation (1). It can be seen at once that if we write

$$
2 d \Sigma_{(1)}=d S^{23}+i d S^{14}, 2 d \Sigma_{(2)}=d S^{31}+i d S^{24}, 2 d \Sigma_{(3)}=d S^{12}+i d S^{34}
$$

then

$$
\left.\begin{array}{l}
d \Sigma_{(1)}=d \Sigma_{(1)}^{\prime} \\
d \Sigma_{(2)}=d \Sigma_{(2)}^{\prime} \cos (\theta+i \phi)-d \Sigma_{(3)}^{\prime} \sin (\theta+i \phi) \\
d \Sigma_{(3)}=d \Sigma_{(3)}^{\prime} \cos (\theta+i \phi)+d \Sigma_{(2)}^{\prime} \sin (\theta+i \phi)
\end{array}\right\} .
$$

There is thus a one-one correspondence between the transformations of the coordinates $x^{\mu}$ which preserve their galilean character, and the transformations of the quantities $d \Sigma$ as cartesian coordinates in complex three-dimensional space.

III. It is convenient to introduce three symbols for tensors which will be used as operators. These are:

and

$$
\begin{aligned}
D_{\rho \sigma}^{\mu \nu} & =\frac{1}{2}\left(g_{\rho}^{\mu} g_{\sigma}^{\nu}-g_{\sigma}^{\mu} g_{\rho}^{\nu}\right), \\
E_{\rho \sigma}^{\mu \nu} & =\frac{1}{2} \sqrt{-g} \epsilon_{\alpha \beta \rho \sigma} g^{\mu \alpha} g^{\nu \beta}, \\
J_{\rho \sigma}^{\mu \nu} & =\frac{1}{2}\left(D_{\rho \sigma}^{\mu \nu}+i E_{\rho \sigma}^{\mu \nu}\right) .
\end{aligned}
$$

These tensors have the following properties which are easily verified.

$$
\begin{aligned}
& E_{\rho \sigma}^{\mu \nu}=-\frac{1}{2} \frac{1}{\sqrt{-g}} \epsilon^{\gamma \delta \mu \nu} g_{\gamma \rho} g_{\delta \sigma} ; \\
& D_{\rho \nu}^{\mu \nu}=3 g_{\rho}^{\mu}, D_{\mu \nu^{\prime}}^{\mu \nu}=6 \\
& E_{\rho \nu}^{\mu \nu}=0 \\
& \Delta_{\rho \nu}^{\mu \nu}=\frac{3}{4} g_{\rho}^{\mu}, \Delta_{\mu \nu}^{\mu \nu}=3 ; \\
& D_{\alpha \beta}^{\mu \nu} D_{\rho \sigma}^{\alpha \beta}=D_{\rho \sigma}^{\mu \nu}, D_{\alpha \beta}^{\mu \nu} E_{\rho \sigma}^{\alpha \beta}=E_{\alpha \beta}^{\mu \nu} D_{\rho \sigma}^{\alpha \beta}=E_{\rho \sigma}^{\mu \nu}, E_{\alpha \beta}^{\mu \nu} E_{\rho \sigma}^{\alpha \beta}=-D_{\rho \sigma}^{\mu \nu} ; \\
& D_{\alpha \beta}^{\mu \nu} \Delta_{\rho \sigma}^{\alpha \beta}=\Delta_{\alpha \beta}^{\mu \nu} D_{\rho \sigma}^{\alpha \beta}=\Delta_{\rho \sigma}^{\mu \nu}, E_{\alpha \beta}^{\mu \nu} \Delta_{\rho \sigma}^{\alpha \beta}=\Delta_{\alpha \beta}^{\mu \nu} E_{\rho \sigma}^{\alpha \beta}=-i \Delta_{\rho \sigma}^{\mu \nu} ; \\
& \Delta_{\alpha \beta}^{\mu \nu} \Delta_{\rho \sigma}^{\alpha \beta}=\Delta_{\rho \sigma}^{\mu \nu} .
\end{aligned}
$$


We now apply these operators to the Riemann-Christoffel tensor, $B_{\mu \nu \rho \sigma}$, whose indices we write in an order such that $B_{\mu \nu \rho \sigma}=-B_{\nu \mu \rho \sigma}=-B_{\mu \nu \sigma \rho}=B_{\rho \sigma \mu \nu} . \quad$ By $B_{\rho \sigma}^{\mu \nu}$ is meant $g^{\mu \alpha} g^{\nu \beta} B_{a \beta \rho \sigma}$.

We have

and

$$
\begin{aligned}
& D_{a \beta}^{\mu \nu} B_{\rho \sigma}^{\alpha \beta}=B_{a \beta}^{\mu \nu} D_{\rho \sigma}^{\alpha \beta}=B_{\rho \sigma}^{\mu \nu}, \\
& E_{a \beta}^{\mu \nu} B_{\rho \nu}^{\alpha \beta}=B_{\alpha \beta}^{\mu \nu} E_{\rho \nu}^{\alpha \beta}=0,
\end{aligned}
$$

the latter result following from the identity

$$
B_{\mu \nu \rho \sigma}+B_{\mu \rho \sigma \nu}+B_{\mu \sigma \nu \rho}=0 .
$$

Let $\Pi_{\rho \sigma}^{\mu \nu}=\Delta_{\alpha \beta}^{\mu \nu} B_{\gamma \delta}^{\alpha \beta} \Delta_{\rho \sigma}^{\gamma \delta}-\frac{1}{6} G . \Delta_{\rho \sigma}^{\mu \nu}, \quad$ where $G_{\rho}^{\mu}=B_{\rho{ }^{\prime}}^{\mu \nu}, G=G_{\mu}^{\mu} . \quad$ It is evident that

$$
\Delta_{\alpha \beta}^{\mu \nu} \Pi_{\rho \sigma}^{\alpha \beta}=\Pi_{\alpha \beta}^{\mu \nu} \Delta_{\rho \sigma}^{\alpha \beta}=\Pi_{\rho \sigma}^{\mu \nu} .
$$

We shall proceed to show that

$$
\Pi_{\rho \nu}^{\mu \nu}=0 .
$$

We have

$$
\Delta_{\alpha \beta}^{\mu \nu} B_{\gamma \delta}^{\alpha \beta} \Delta_{\rho \sigma}^{\gamma \delta}=\frac{1}{4}\left(B_{\rho \sigma}^{\mu \nu}-E_{\alpha \beta}^{\mu \nu} B_{\gamma \delta}^{\alpha \beta} E_{\rho \sigma}^{\gamma \delta}\right)+i\left(E_{\alpha \beta}^{\mu \nu} B_{\rho \sigma}^{\alpha \beta}+B_{a \beta}^{\mu \nu} E_{\rho \sigma}^{\alpha \beta}\right) .
$$

The imaginary part of this gives zero when contracted; to evaluate the real part we take the term

$$
-E_{a \beta}^{\mu \nu} B_{\gamma \delta}^{\alpha \beta} E_{\rho \sigma}^{\gamma \delta}=\frac{1}{4} \epsilon^{\mu \nu a \beta} \epsilon_{\rho \sigma \gamma \delta} B_{a \beta}^{\gamma \delta} .
$$

Now

$$
\frac{1}{4} \epsilon^{\mu \nu \alpha \beta} \epsilon_{\rho \sigma \gamma \delta}=D_{\rho \sigma}^{\mu \nu} D_{\gamma \delta}^{\alpha \beta}+D_{\rho \gamma}^{\mu \nu} D_{\delta \sigma}^{\alpha \beta}+D_{\rho \delta}^{\mu \nu} D_{\sigma \gamma}^{\alpha \beta}+D_{\gamma \delta}^{\mu \nu} D_{\rho \sigma}^{\alpha \beta}+D_{\delta \sigma}^{\mu \nu} D_{\rho \gamma}^{\alpha \beta}+D_{\sigma \gamma}^{\mu \nu} D_{\rho \delta}^{\alpha \beta} .
$$

This is in fact nothing else than the Laplace rule of expansion of a determinant of the fourth order in terms of minors of the second order.

We have thus

$$
\begin{aligned}
-E_{\alpha \beta}^{\mu \nu} B_{\gamma \delta}^{\alpha \beta} E_{\rho \sigma}^{\gamma \delta} & =B_{\rho \sigma}^{\mu \nu}-2 D_{\rho \gamma}^{\mu \nu} G_{\sigma}^{\gamma}+2 D_{\sigma \gamma}^{\mu \nu} G_{\rho}^{\gamma}+D_{\rho \sigma}^{\mu \nu} \cdot G \\
& =B_{\rho \sigma}^{\mu \nu}-\underline{a}_{\hat{r}}^{\mu} G_{\sigma}^{\nu}+g_{\rho}^{\nu} G_{\sigma}^{\mu}+g_{\sigma}^{\mu} G_{\rho}^{\nu}-g_{\sigma}^{\nu} G_{\rho}^{\mu}+D_{\rho \sigma}^{\mu \nu} \cdot G,
\end{aligned}
$$

whence

$$
\Pi_{\rho \nu}^{\mu \nu}=\frac{1}{4}\left(2 G_{\rho}^{\mu}-g_{\rho}^{\mu} G+G_{\rho}^{\mu}+G_{\rho}^{\mu}-4 G_{\rho}^{\mu}+\frac{33}{2} g_{\rho}^{\mu} G\right)-\frac{1}{6} \frac{3}{4} g_{\rho}^{\mu} G=0 .
$$

IV. Consider now the invariant

$$
\Pi_{\mu \nu \rho \sigma} d S^{\mu \nu} d S^{\rho \sigma}=\Pi_{\mu \nu \rho \sigma} d \Sigma^{\mu \nu} d \Sigma^{\rho \sigma}
$$

where $d \sum^{\mu \nu}=\Delta_{a \beta}^{\mu \nu} d S^{\alpha \beta}$. 
It is easy to see that in galilean coordinates the relations (2) and (3) satisfied by $\Pi_{\mu \nu \rho \sigma}$ become

$$
\begin{aligned}
& \qquad \Pi_{2323}=-\Pi_{1414}=-i \Pi_{1423} \text {, etc., } \\
& \text { together with } \Pi_{2323}+\Pi_{3131}+\Pi_{1212}=0 .
\end{aligned}
$$

So that $\Pi_{\mu \nu \rho \sigma} d S^{\mu \nu} d S^{\rho \sigma}$ becomes 16 times the ternary quadratic

$$
\begin{gathered}
\Pi_{2323} d \Sigma_{(1)}^{2}+\Pi_{3131} d \Sigma_{(2)}^{2}+\Pi_{1212} d \Sigma_{(3)}^{2} \\
+2 \Pi_{3112} d \Sigma_{(2)} d \Sigma_{(3)}+2 \Pi_{1223} d \Sigma_{(3)} d \Sigma_{(1)}+2 \Pi_{2331} d \Sigma_{(1)} d \Sigma_{(2)} .
\end{gathered}
$$

The proposed new method of fixing the directions of the axes is that of transforming the surface variables so that this quadratic becomes a sum of squares. With such a transformation is associated uniquely a transformation of the point-coordinates, as shown in section II, so that the axes can always be chosen to fulfil this condition, and uniquely if the roots of the discriminating cubic of (5) are all different.

Moreover if we write $\Pi_{\rho \sigma}^{\mu \nu}=\frac{1}{2}\left(P_{\rho \sigma}^{\mu \nu}+i Q_{\rho \sigma}^{\mu \nu}\right)$ then

$$
B_{\mu \nu \rho \sigma}=P_{\mu \nu \rho \sigma}+\frac{1}{2} g_{\mu \rho} G_{\nu \sigma}+\frac{1}{2} g_{\nu \sigma} G_{\mu \rho}-\frac{1}{2} g_{\mu \sigma} G_{\nu \rho}-\frac{1}{2} g_{\nu \rho} G_{\mu \sigma}-{ }_{: 3}^{1} D_{\mu \nu \rho \sigma} G .
$$

The $B_{\mu \nu \rho \sigma}$ are thus expressible uniquely in terms of the $P_{\mu \nu \rho \sigma}$ and the $G_{\mu \nu}$. The equations (4) show that there are exactly 10 independent $P_{\mu \nu \rho \sigma}$. The numerical values of the $P_{\mu r \rho \sigma}$ and the $G_{\mu \nu}$ at any one point are thus independent of one another, and the use of the $\Pi_{\mu \nu \rho \sigma}$ to fix the directions of the axes will not be affected by the degeneracy of $G_{\mu \nu} d x^{\mu} d x^{\prime}$.

V. The coefficients of the discriminating cubic of (5) are given by the four invariants $P_{\rho \sigma}^{\mu l} P_{\mu \nu^{\prime}}^{\rho \sigma}, P_{\rho \sigma}^{\mu \nu} Q_{\mu \nu^{\prime}}^{\rho \sigma}, P_{\rho \sigma}^{\mu l} P_{\tau \nu}^{\rho \sigma} P_{\mu \nu}^{\tau v}, P_{\rho \sigma}^{\mu l} P_{\tau v}^{\rho \sigma} Q_{\mu \nu}^{\tau v}$. These form a complete system for invariants which are functions of the $P$ 's and $Q$ 's alone.

The total number of algebraically independent invariants of the $B_{\mu r \cdot \rho \sigma}$ is 14 . Four of these are expressible in terms of the $G$ 's alone and four in terms of the $P$ 's alone. The remaining six require both $G$ 's and $P$ 's to express them and give the coefficients of the transformation from one set of absolute axes to the other.

For the field of an isolated particle with the metric

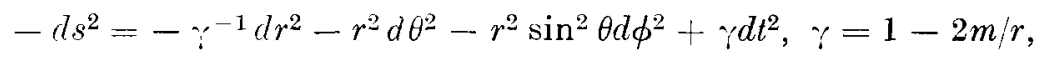

the components of $P$ are:

$$
P_{23}^{2: 3}=P_{14}^{14}=-\frac{2 m}{r^{3}}, P_{31}^{31}=P_{24}^{24}=P_{12}^{12}=P_{34}^{34}=\frac{m}{r^{3}},
$$

the remaining effectively different components vanishing. 
The axial directions are thus already principal directions in our sense. But this property will be preserved if the axes are rotated arbitrarily in the $\theta$ plane, or set in motion in the $r$ direction. This degeneracy of the field is expressed in terms of the invariants by the vanishing of $P_{\rho \sigma}^{\mu \nu} Q_{\mu \nu}^{\rho \sigma}$ and $P_{\rho \sigma}^{\mu v} P_{\tau \nu}^{\rho \sigma} Q_{\mu \nu}^{\tau v}$ and by the relation $\left(P_{\rho \sigma}^{\mu \cdot} P_{\mu \nu}^{\rho \sigma}\right)^{3}=\left(\begin{array}{lll}P_{\rho \sigma}^{\mu \nu} & P_{\tau \nu}^{\rho \sigma} P_{\mu \nu}^{\tau \nu}\end{array}\right)^{2}$. This example raises the problem of determining just what relations between the invariants correspond to the properties of being symmetrical, being static and so on.

For a gravitational field which is not further degenerate the four $P$ invariants will be independent functions of the coordinates. Some set of four standard functions of the invariants can thus be taken as a system of canonical coordinates for all fields of this type. The known solutions of Einstein's equations have all, unfortunately, properties of symmetry which prevent this procedure being applied to them.

VI. It may be of interest to note that we can use the operator $\Delta_{\rho \sigma}^{\mu \nu}$ to express Maxwell's equations in the simple form

$$
\Phi_{, v}^{\mu \nu}=J^{\mu}
$$

where $J_{\mu}$ is the charge-and-current vector, $F_{\mu \nu}$ the electromagnetic force tensor, and $\Phi_{\mu \nu}=\Delta_{\mu \nu}^{\rho \sigma} F_{\rho \sigma}^{\prime}$.

\section{King's College,}

Cambridge. 